\title{
Aspectos metodológicos do Estudo Nacional de Alimentação e Nutrição Infantil (ENANI-2019)
}

Sabemos que a prevalência da desnutrição infantil no Brasil, pelo indicador altura para idade, diminuiu de 13,5\%, em 1996, para 6,8\%, em 2006/2007, em menores de cinco anos 1. Conhecemos que a prevalência de aleitamento materno exclusivo aos seis meses teve tendência ascendente, de 4,7\%, em 1986, para 37,1\%, em 2006, tendo praticamente se estabilizado em 2013, quando alcançou 36,6\% 2. Temos ciência de que a prevalência de anemia era de 20,9\% e a deficiência de vitamina A atingia 17,4\% das crianças de 6 a 59 meses em 2006 3. Temos informações de que 32,3\% das crianças menores de dois anos consumiam refrigerantes e 60,8\% ingeriam biscoitos ou doces em 2013 4. A partir daí não temos mais estimativas nacionais a respeito desses importantes indicadores. Essa situação de desconhecimento mudou com a realização do Estudo Nacional de Alimentação e Nutrição Infantil (ENANI-2019), no qual foram estudadas 14.558 crianças menores de cinco anos, de março de 2019 a março de 2020. Por meio desse importante estudo teremos, em breve, informações atualizadas a respeitos de vários indicadores de alimentação e nutrição infantis.

Neste fascículo, CSP publica cinco artigos descrevendo os aspectos metodológicos do ENANI-2019 5,6,7,8,9. A pesquisa investigou três componentes: aleitamento e consumo alimentar, estado nutricional por meio de antropometria e deficiências de vários micronutrientes - ferro (hemoglobina e ferritina), zinco, selênio, ácido fólico, vitaminas A, B1, B6, B12, D e E. O último estudo nacional sobre saúde e nutrição de crianças foi realizado há mais de dez anos, em 2006, que, dentre as deficiências de micronutrientes, investigou apenas carências de ferro e vitamina A1. O ENANI-2019 é mais abrangente na investigação dessas deficiências.

O leitor vai encontrar nesses artigos descrições bem detalhadas dos métodos utilizados no estudo, envolvendo amostragem, avaliação do consumo alimentar, antropométrica e de micronutrientes. No estudo foram aplicadas as mais modernas técnicas de amostragem e de análise de desenhos complexos de amostragem: estratificação por macrorregião, conglomeração por setor censitário, amostragem inversa, ponderação e calibração. Apesar da interrupção prematura do estudo por causa da pandemia da COVID-19, quando foram atingidos $83,5 \%$ da amostra pretendida, o grande tamanho amostral alcançado permitirá que estimativas nacionais e por macrorregiões geográficas sejam produzidas com pequena
1 Departamento de Saúde Pública, Universidade Federal do Maranhão, São Luís, Brasil. 
margem de erro. A taxa de recusas no ENANI-2019 foi de 35,8\%, dentro do esperado nos estudos conduzidos nos tempos atuais, nos quais as taxas de recusa têm sido elevadas e crescentes. É triste constatar que vários setores censitários tiveram que ser substituídos em função da violência em áreas dominadas pelo tráfico de drogas e devido a fake news, que espalharam a ideia de que a intenção do estudo era de transmitir doenças infecciosas e de raptar crianças para o roubo de órgãos.

O estudo conseguiu obter amostras de sangue venoso de 70\% das crianças elegíveis de 6 a 59 meses e teve uma logística complexa de coleta, transporte e análise padronizada dos exames laboratoriais. Interessante ressaltar a parceria público-privada realizada no estudo, no qual a rede de laboratórios Diagnósticos do Brasil participou da coleta e análise de espécimes laboratoriais, a Science (Sociedade para o Desenvolvimento da Pesquisa Científica) colaborou na realização do processo de amostragem e na coleta de dados, e a InMedia Comunicação ajudou na interação entre os pesquisadores com governos, profissionais de saúde e com os demais veículos de comunicação.

O consumo alimentar foi investigado por meio de questionário e recordatório alimentar de 24 horas em praticamente todas as crianças da amostra. A coleta dos dados de consumo alimentar foi realizada de forma padronizada por meio de aplicativo (24HR-App) e manual fotográfico, especialmente desenvolvidos para o ENANI-2019. Uma limitação foi que não houve repetição do inquérito alimentar recordatório de 24 horas para que se pudesse corrigir a variabilidade interpessoal com dados do estudo.

Na parte antropométrica, foram utilizados, de forma cuidadosa, os procedimentos padronizados mais recomendados para mensurar peso e altura de crianças. Foram utilizadas balanças e antropômetros portáteis, a medida do peso foi realizada por duas e a da altura por três pessoas, para controle de qualidade.

A comunidade científica e os gestores estão ansiosos para conhecer os resultados do ENANI-2019. Será muito importante saber as tendências que os indicadores de alimentação e nutrição das crianças menores de cinco anos tiveram nos últimos 16 anos. Várias questões poderão ser respondidas. O declínio na prevalência de desnutrição infantil continuou? Houve aumento na prevalência de obesidade? As prevalências de aleitamento materno aumentaram ou continuaram estabilizadas? Houve redução nas prevalências de anemia e da deficiência de vitamina A? Qual a prevalência atual de várias deficiências de micronutrientes para as quais ainda não havia estimativas nacionais? Ademais, o estudo nos permitirá acompanhar a evolução das desigualdades regionais e socioeconômicas em relação a todos esses indicadores e saber se as desigualdades estão diminuindo, estáveis ou aumentando. Os conhecimentos gerados pelo ENANI-2019 serão essenciais para reorientar as políticas públicas de alimentação e nutrição infantil nos anos vindouros. 


\section{Informação adicional}

ORCID: Antônio Augusto Moura da Silva (00000003-4968-5138).

1. Monteiro CA, Benicio MHD’A, Konno SC, Silva, ACF, Lima ALL, Conde WL. Causas do declínio da desnutrição no Brasil. Rev Saúde Pública 2009; 43:35-43.

2. Boccolini CS, Boccolini PMM, Monteiro FR, Venâncio SI, Giugliani EJ. Tendência de indicadores de aleitamento materno no Brasil em três décadas. Rev Saúde Pública 2017; 51:108.

3. Ministério da Saúde. Pesquisa Nacional de Demografia e Saúde da Criança e da Mulher PNDS 2006: dimensões do processo reprodutivo e da saúde da criança. Brasília: Ministério da Saúde; 2009.

4. Jaime PC, Frias PG, Monteiro HOC, Almeida PVB, Malta DC. Healthcare and unhealthy eating among children aged under two years: data from the National Health Survey, Brazil, 2013. Rev Bras Saúde Matern Infant 2016; 16:14957.

5. Alves-Santos NH, Castro IRR, Anjos LA, Lacerda EMA, Normando P, Freitas MB, et al. General methodological aspects in the Brazilian National Survey on Child Nutrition (ENANI-2019): a population-based household survey. Cad Saúde Pública 2021; 37:e00300020.
6. Lacerda EMA, Boccolini CS, Alves-Santos NH, Castro IRR, Anjos LA, Crispim SP, et al. Methodological aspects of the assessment of dietary intake in the Brazilian National Survey on Child Nutrition (ENANI-2019): a population-based household survey. Cad Saúde Pública 2021; 37:e00301420.

7. Anjos LA, Ferreira HS, Alves-Santos NH, Freitas MB, Boccolini CS, Lacerda EMA, et al. Methodological aspects of the anthropometric assessment in the Brazilian National Survey on Child Nutrition (ENANI-2019): a populationbased household survey. Cad Saúde Pública 2021; 37:e00293320.

8. Castro IRR, Normando P, Alves-Santos NH, Bezerra FF, Reis MC, Pedrosa LFC, et al. Methodological aspects of the micronutrient assessment in the Brazilian National Survey on Child Nutrition (ENANI-2019): a populationbased household survey. Cad Saúde Pública 2021; 37:e00301120.

9. Vasconcellos MTL, Silva PLN, Castro IRR, Boccolini CS, Alves-Santos NH, Kac G. Sampling plan of the Brazilian National Survey on Child Nutrition (ENANI-2019): a populationbased household survey. Cad Saúde Pública 2021; 37:e0037221. 